Cahiers $d u$ MONDE RUSSE

\section{Cahiers du monde russe}

Russie - Empire russe - Union soviétique et États indépendants

$51 / 1 \mid 2010$

Pierre le Grand et ses images de Rome

\title{
De Re Militari dans la bibliothèque de Pierre le Grand
}

Military science books from Peter the Great's library

\section{Émilie d'Orgeix}

\section{(2) OpenEdition}

Journals

Édition électronique

URL : https://journals.openedition.org/monderusse/9177

DOI : 10.4000/monderusse. 9177

ISSN : $1777-5388$

Éditeur

Éditions de l'EHESS

Édition imprimée

Date de publication : 15 avril 2010

Pagination : 121-133

ISBN : 978-2-7132-2306-8

ISSN : $1252-6576$

Référence électronique

Émilie d'Orgeix, «De Re Militari dans la bibliothèque de Pierre le Grand », Cahiers du monde russe [En ligne], 51/1 | 2010, mis en ligne le 10 mai 2013, consulté le 04 septembre 2022. URL : http:// journals.openedition.org/monderusse/9177; DOI : https://doi.org/10.4000/monderusse. 9177

Ce document a été généré automatiquement le 4 septembre 2022

Tous droits réservés 


\title{
De Re Militari dans la bibliothèque de Pierre le Grand
}

\author{
Military science books from Peter the Great's library
}

\author{
Émilie d'Orgeix
}

1 Dès le $\mathrm{xvI}^{\mathrm{e}}$ siècle, la vitalité de l'édition militaire, nourrie par la querelle des lettres et des armes et l'abondante production des soldats-lettrés, engage tout prince éclairé à doter sa bibliothèque d'un imposant rayonnage de manuscrits et d'imprimés consacré aux écrits militaires. Histoire, biographie, stratégie, sciences, inventions, technique d'attaque et défense des places constituent ces ensembles qui se fondent, comme dans le domaine des arts et les lettres, sur l'héritage antique. La constitution de ces volumineuses bibliothèques militaires est guidée par les recommandations d'auteurs dont Roberto Valturio, le premier, donne en 1470 une longue liste alphabétique des ouvrages consultés pour rédiger son traité latin De Re Militari ${ }^{1}$. Si la présence des auteurs antiques est encore écrasante dans sa bibliographie, l'union de l'art de la guerre antique et de l'artillerie moderne va rapidement accroître le nombre des écrits consacrés aux travaux de Mars. Composée à l'origine de livres antiques d'historiographie, de biographie et de stratégie, elle s'enrichit progressivement d'essais de penseurs et de praticiens de l'art de la guerre plus récents comme Machiavel, Montecuccoli ou Du Bellay ainsi que d'une multitude de traités enseignant l'art de la fortification moderne ${ }^{2}$. Alors que les princes condottieres de la fin du $\mathrm{xv}^{\mathrm{e}}$ siècle, tel Antonio da Marciano ${ }^{3}$, pouvaient encore harmonieusement équilibrer culture militaire antique et essais modernes dans leurs bibliothèques, les questions de choix et de sélection des ouvrages, classiques et contemporains, nationaux et étrangers, littéraires et techniques, théoriques et pratiques, deviennent essentielles un siècle plus tard. Le genre historique et biographique antique, modèle par excellence du soldat humaniste, s'enrichit d'un corpus théorique et pratique qui concurrence, et parfois même contredit, les vertus guerrières au profit de sciences et techniques nouvelles.

2 De fait, la composition de la bibliothèque du prince moderne, miroir de ses penchants, de ses aspirations sociales et politiques, de son dilettantisme, de sa volonté de spécialisation, de son intérêt pour l'actualité, trouve un écho particulier dans le choix de 
ses ouvrages militaires. Si, pour citer Francesco Patrizi, «l'art militaire comme ses sœurs et ses servantes, l'architecture, la sculpture, la peinture, la métallurgie, la musique, la médecine, la philosophie, la mathématique, la mécanique, s'apprennent toutes dans et par les livres ", les objections selon lesquelles les livres militaires ne sont pas à même d'enseigner «l'art de combattre » ne sont pas valides ${ }^{4}$. L'équilibre entre auteurs anciens et modernes, entre poliorcétique antique et architecture bastionnée, révèle plus encore que le goût de leur propriétaire, l'expression de sa modernité.

Dans le contexte du règne de Pierre le Grand, les sources littéraires de la "révolution » militaire russe ont nourri en première ligne le feu des réformes pétroviennes dont la centralisation des pouvoirs, la réorganisation et la modernisation des armées et l'éducation des élites militaires ${ }^{5}$. De fait, la collection de militaria de la bibliothèque de Pierre le Grand représente un cas d'étude particulièrement intéressant. Elle permet d'éclairer l'attitude d'un souverain, féru d'art et d'antiquités, envers l'art militaire et de découvrir les lectures qu'il a privilégiées et les guides littéraires qui ont accompagné la construction ex nihilo de la plus grande ville fortifiée du XvIII ${ }^{e}$ siècle dans un processus historique unique de création d'un Empire dans un monde traversé par le grand bouleversement baroque.

\section{Pierre le Grand et sa bibliothèque : sources et constitution}

4 Avant de s'engager dans l'analyse des sources, il convient cependant de souligner combien cette entreprise doit être conduite avec prudence et méthode. En premier lieu, car l'histoire même de la constitution de la collection, qui a récemment fait l'objet de plusieurs études, a été caractérisée par une politique d'acquisition intense et cumulative $^{6}$. Souverain épris de connaissances et de possessions, Pierre le Grand n'a pas échappé à la passion compulsive des grands collectionneurs. Entre 1713 et 1724, il requiert de ses agents placés dans toutes les grandes villes d'Europe qu'ils lui fassent parvenir tous les documents manuscrits et imprimés d'art et d'architecture civile et militaire qu'ils pourraient se procurer. L'ensemble résultant de ces achats, dons et parfois même spoliations permet de comprendre la composition à la fois lacunaire et redondante d'une collection dont les titres glanés à travers toute l'Europe n'ont pas été compilés en achats coordonnés. En second lieu, car la bibliothèque de Pierre le Grand n'a pas été rassemblée dès son origine dans un lieu dédié. Conçue comme une documentation itinérante, transportée dans des caisses au gré des séjours du tsar dans ses résidences, elle connut plusieurs lieux temporaires avant d'être réunie à partir de 1719 dans une kunstkamera selon un projet de Georg Johann Mattarnovi. Une grande partie de ce fonds originel est aujourd'hui conservée à la bibliothèque de l'Académie des sciences de SaintPétersbourg7. Néanmoins, la collection actuelle ne rassemble pas l'intégralité des ouvrages acquis par Pierre le Grand. La bibliothèque fut distraite au cours du XVIII siècle avant d'être reconstituée dans les fonds de l'Académie des sciences de Saint-Pétersbourg (BAN) où elle fut fondue avec l'ensemble des collections patrimoniales. C'est seulement au milieu du $\mathrm{xx}^{\mathrm{e}}$ siècle qu'un patient travail d'inventaire a permis d'isoler et d'établir une liste des ouvrages provenant de la collection de Pierre le Grand et de son fils Aleksej. Ce catalogue n'étant ni achevé ni exhaustif, l'analyse des sources requiert une méthode particulière, qui ne peut être que partielle et réalisée « en positif ", c'est-à-dire à partir des exemplaires existants, puisque la démarche opposée ne pourrait correspondre qu'à 
une section distraite de la bibliothèque dont on découvrira peut-être un jour l'existence. Ainsi, certains ouvrages aujourd'hui lacunaires, telle la traduction des Stratagèmes de Sextus Julius Frontin que Pierre le Grand avait commandée dès 1692, n’y apparaît pas. On peut pourtant penser que cette traduction, préparée par Karion Istomin - Kniga $o$ hitrostjah ratnyh - était présente dans sa bibliothèque ${ }^{8}$.

5 C'est donc une lecture des sources à la fois circonspecte et opportuniste que ce présent article livre pour illustrer la disposition de Pierre le Grand envers l'art militaire antique et moderne. Elle s'appuie sur l'analyse des titres présents dans le catalogue des manuscrits de la bibliothèque de Pierre le Grand, que l'on doit à Irina Lebedeva, ${ }^{9}$ ainsi que celui des imprimés que prépare actuellement Irina Hmelevskih.

\section{Pierre le Grand et l'art militaire des anciens}

6 S'il n'existe d'autres inventaires d'auteurs antiques que ceux donnés dans les bibliographies des traités militaires, trois types de production éditoriale prédominent dans les écrits antiques : biographique, historiographique et scientifico-technique. Parmi les premiers, on compte des ouvrages récurrents dans les bibliothèques tels De vita duodecim Caesarum Libri et De Viris Illustribus de Suétone, Historiae de son contemporain

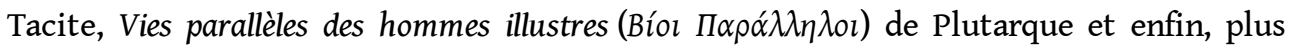
rarement, les maximes historiques de Valère Maxime (Factorum dictorumque memorabilium libri IX). Parmi les ouvrages d'histoire, hormis le grand best-seller que constitue Commentarii Rerum Gestarum (comprenant De Bello Gallico et De Bello Civile) de Jules César, on trouve aussi les écrits de Salluste (Historiae et Bellum Jugurthinum), de TiteLive (Ab Urbe Condita), de Tacite (Historiae et De Vita Agricolae) et de Flavius Josèphe (Bellum Judaicum). L'art militaire, qui englobe tant la tactique et la stratégie grecque et romaine que les écrits techniques, conjugue non seulement des essais de castramétation, de mouvements des troupes et d'organisation des légions comme De Re Militari de Flavius Végèce, De Romanorum militia et castrorum metatione liber de Polybe mais également de nombreux ouvrages valorisant la force physique, voire l'athlétisme militaire ainsi que la conduite militaire et l'ingéniosité humaine à travers des publications telles que De stratagemis de Polyen ou Stratagemi militari et Astutie militari de Sextus Julius Frontin.

7 L'ensemble de ces écrits antiques, rédigés dans les formes du dialogue, de la relation épistolaire et des maximes, a été fondamental pour forger en Europe un code humaniste de valeurs militaires, alternatif au code chevaleresque et transfiguré par le culte de l'Antiquité, à partir du $\mathrm{XVI}^{\mathrm{e}}$ siècle $^{10}$. C'est grâce à leur diffusion et à leurs nombreuses éditions commentées et rééditions en latin et en langue vulgaire que la profession militaire s'intellectualise par l'union des armes et des lettres. C'est aussi grâce à leur relecture et leur interprétation que la figure du prince guerrier trouvera toute sa place dans les écrits renaissants.

8 Or, la lecture du catalogue des ouvrages de Pierre le Grand est à première vue surprenante, si l'on considère le peu de titres consacrés à l'art militaire antique. Sa fascination pour les exploits militaires antiques est pourtant documentée. Avram Veselovskij, résident russe en Autriche, lui envoie une lettre en 1716 à propos de l'achat d'un "Abrégé des actions militaires de Jules César »"11 en latin que Pierre le Grand lui commande :

J'ai reçu l'ordre de Votre Majesté, envoyé de Gdansk le 28 mars, dans lequel vous m'ordonnez de trouver le livre abrégé des actions militaires de Jules César en 
langue latine, de l'acheter et de l'envoyer immédiatement à Votre Majesté. J'ai essayé de trouver ce livre, non seulement ici mais aussi à Leipzig, mais je ne l'ai trouvé qu'en français - version que je peux avoir - car la latine n'est jamais parue. En revanche ce qui existe c'est la Vie complète de Jules César en latin et si Votre Majesté désire, je pourrais le lui acheter et envoyer, de même que le livre désiré en français, car ce dernier je le possède déjà. ${ }^{12}$

La mention de la langue latine est en soi intéressante. Elle démontre la culture classique de Pierre le Grand qui préférait, au premier chef, les éditions latines plutôt que leurs traductions. Le titre demandé est également révélateur. Ce sont tout naturellement les ouvrages biographiques mettant en valeur les actions militaires des grands empereurs qui intéressent Pierre le Grand, dont la Vie d'Alexandre (Aleksandrika) ${ }^{13}$, l'un des livres lus dès son enfance et dont la traduction en russe devait paraître à Amsterdam ${ }^{14}$. Enfin, la version « abrégée » est également révélatrice de la personnalité du tsar qui, en prince lettré, ne disposait que de peu de temps pour ses lectures et favorisait les versions succinctes. De fait, les œuvres de Jules César sont les mieux représentées dans la bibliothèque de Pierre. Il y apparait à deux reprises à travers une édition de Commentarii accompagnée de ses annexes par Aulus Hirtius, légat de l'Empereur en Gaule qui avait souvent été chargé de négociations délicates ${ }^{15}$. Dans le même ordre d'idées, les hauts faits d'Alexandre le Grand l'intéressent au premier chef. On trouve ainsi un exemplaire de l'ouvrage de Quintus Curtius Rufus, Historiae Alexandri Magni, mais cette fois-ci traduit et conservé dans la partie russe du fonds de la bibliothèque. Selon le témoignage du captif suédois, Lars Johan Ehrenmalm, Pierre le Grand « ordonna de traduire en russe les livres de Jules César et de Curtius, qu'il consulte lui-même [...] ${ }^{16}$.

Néanmoins, le complément de la bibliothèque est plus lacunaire. Tacite est, par la suite, mentionné par un petit texte historique et ethnographique consacré aux us et coutumes des Germains intitulé De situ ac populis Germaniae. Flavius Josèphe y est présent par un manuscrit de La Guerre des juifs, ouvrage qui fut traduit en russe dès le xII ${ }^{\mathrm{e}}$ siècle et cité comme modèle de "l'écriture guerrière $»^{17}$. Enfin, on y trouve aussi l'opuscule d'Aélien De militaribus ordinibus sur l'organisation de l'armée grecque ${ }^{18}$ [ill.1]. Mais, si l'on considère la richesse de la littérature antique, une telle sous-représentation - même si l'on pense aux historiens classiques dont les textes faisaient partie de la «bibliothèque historique » orthodoxe que Pierre hérita de ses ancêtres - est étonnante d'autant plus que l'on n'y trouve aucun ouvrage d'art militaire tels ceux de Végèce, Polybe ou Frontin. 


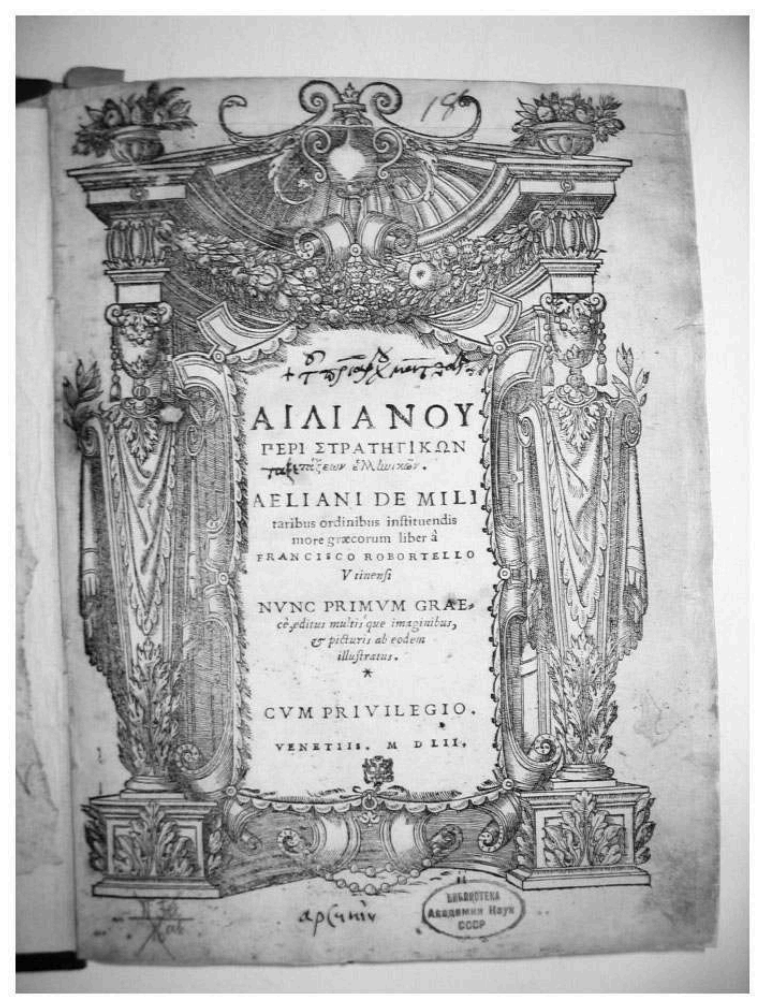

Frontispice du traité d'Aélien, De militaribus ordinibus instituendis more græcorum liber à Francisco Robortello, Venise : Andrea et Jacobum Spinellos, 1552, BAN NIORK, 8011 q/338R.

Cette quasi absence d'ouvrages militaires antiques ouvre la voie à deux interprétations. La première, qu'il est prudent de ne pas écarter, serait que l'ensemble de ces ouvrages qui forment un corpus cohérent, ait été dispersé avant la rédaction des catalogues actuels. La seconde, plus hasardeuse mais également plus fructueuse, appuierait l'hypothèse selon laquelle Pierre le Grand, en véritable homme de son temps, n'aurait volontairement conservé dans sa bibliothèque que des ouvrages militaires qui lui semblaient utiles à son entreprise. Elle conforterait la thèse d'un souverain résolument contemporain dans ses choix, pour lequel architecture militaire rimerait avec modernité.

\section{Pierre le Grand et la modernité}

Cette seconde piste, considérée dans le contexte particulier du début du XVIII siècle, est intéressante à approfondir. En effet, la lecture des traités d'art militaire et de fortification antique avait déjà commencé, à cette époque, à être envisagée de manière accessoire par rapport aux traités consacrés aux nouvelles catégories professionnelles comme les ingénieurs, artilleurs, sapeurs et mineurs. Face à la multiplication des sciences et des écrits sur le génie militaire, que Vauban avait parfaitement résumé en 1670 comme étant « un art qui embrasse trop de matières pour qu'un homme puisse le posséder ", des choix s'imposent. Or, à partir de la seconde moitié du XVII esiècle, la lecture des auteurs antiques se déprécie ouvertement au profit de la lecture d'ouvrages modernes dont les chapitres historiques procurent une connaissance générale. Ainsi, Sébastien le Prestre de Vauban et Alain Manesson Mallet, deux auteurs traduits en russe et imprimés sous les presses de Saint-Pétersbourg au début du xvIII siècle, 
recommandent une étude résolument contemporaine de l'art militaire. Vauban, le premier, dont le traité fut traduit en 1724 à Saint-Pétersbourg par Vasilij Suvorov (1705-1775), personnage très peu sympathique et futur persécuteur des princes Dolgorukij, qui était à l'époque ordonnance et traducteur du tsar, mentionne que «la lecture des auteurs anciens est importante mais qu'il vaut mieux lire des modernes qui traitent également de l'origine de la fortification sans s'embarrasser de détails inutiles $»^{19}$. Alain Manesson Mallet, le second, dont l'ouvrage Les travaux de Mars ou l'art

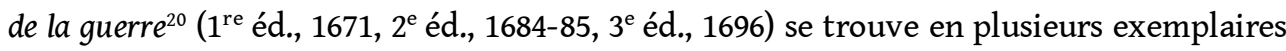
dans la bibliothèque de Pierre le Grand et qui est l'un des premiers auteurs à avoir été traduits en russe en 1720 , adopte le même discours, critiquant notamment l'ouvrage de Mathias Dögen auteur d'un «si gros volume sur la fortification, que beaucoup de gens l'ont trouvé fort ennuyeux, surtout les citations continuelles sur l'histoire ancienne [qui] ont épuisé la patience de ceux qui l'ont voulu lire». Il corrige également certains modèles de Francesco de Marchi en notant

je m'étonnerais qu'un homme aussi habile que le capitaine de Marchi eut avancé cette proposition, si pour l'excuser, je ne songeais qu'il y a près de cent ans qu'il a écrit et qu'une infinité de sièges et d'expérience nous ont donné des lumières qu'il ne pouvait avoir ${ }^{21}$.

13 Les propos de Vauban et de Manesson Mallet ne sont pas isolés. À partir du début du $\mathrm{XVIII}^{\mathrm{e}}$ siècle, le culte des auteurs antiques s'arrête soudain à l'orée du champ de bataille. Le chevalier de Folard, contemporain de Pierre le Grand, critique ainsi dans ses Commentaires sur Polybe (1724-1730) la stratégie adoptée par le maréchal de Berwick devant Philipsbourg:

L'inventeur de ce beau dessein avait lu sans jugement les Commentaires de César, car le siège d'Alésia de ce Romain et celui de Philipsbourg sont deux choses différentes [...]. C'est tout autre chose aujourd'hui, il faut considérer les temps. ${ }^{22}$

Or, « considérer les temps » semble être très exactement l'attitude qu'adopte Pierre le Grand envers la littérature militaire. Sa collection de militaria est révélatrice de son goût pour les « révolutions présentes ». Si l'on trouve quelques ouvrages très divers datant du début $d u \mathrm{XVII}^{\mathrm{e}}$ siècle, qui paraissent un peu démodés en terme de poliorcétique, comme Buonaiuto Lorini ${ }^{23}$, Wilhelm Dilich ${ }^{24}$, Adam Freitag ${ }^{25}$, Ambroise Bachot ${ }^{26}$, Georg Andreas Böckler $^{27}$, Wendelin Schildknecht ${ }^{28}$, Andreas Cellarius ${ }^{29}$ [ill. 2] ou Antoine Deville ${ }^{30}$, les éditions anciennes sont rares dans les rayonnages de Pierre le Grand. Sa bibliothèque est résolument contemporaine et même d'une étonnante actualité. On y trouve les principaux best-sellers de la fin du XVII siècle, tel L'Art de jetter les bombes de François Blondel $^{31}$ dont un traité fut traduit en russe en 1720 [ill. 3 et 4 ] avec néanmoins une nette prédilection pour les modèles prônés en Europe du Nord, tels ceux de Coehorn ${ }^{32}$, de Werdmüller (traducteur d'Antoine Deville) ${ }^{33}$ ou de Scheiter ${ }^{34}$ ainsi qu'une véritable cohorte d'ouvrages publiés entre 1700 et 1720 durant la période même de constitution de la bibliothèque. Ainsi, Pierre le Grand possède des exemplaires des Mémoires d'artillerie de Saint-Rémy $(1702)^{35}$, de L'architecture militaire et de La Forge de Vulcain de Saint-Julien (1705 et 1706) ${ }^{36}$, des Mémoires pour l'attaque et la défense de Goulon ${ }^{37}$ (1706), de L'Architecture militaire (1702) et Le véritable Vauban (1706) de Sturm ${ }^{38}$ ainsi que plusieurs atlas contemporains des places fortes européennes ${ }^{39}$. 


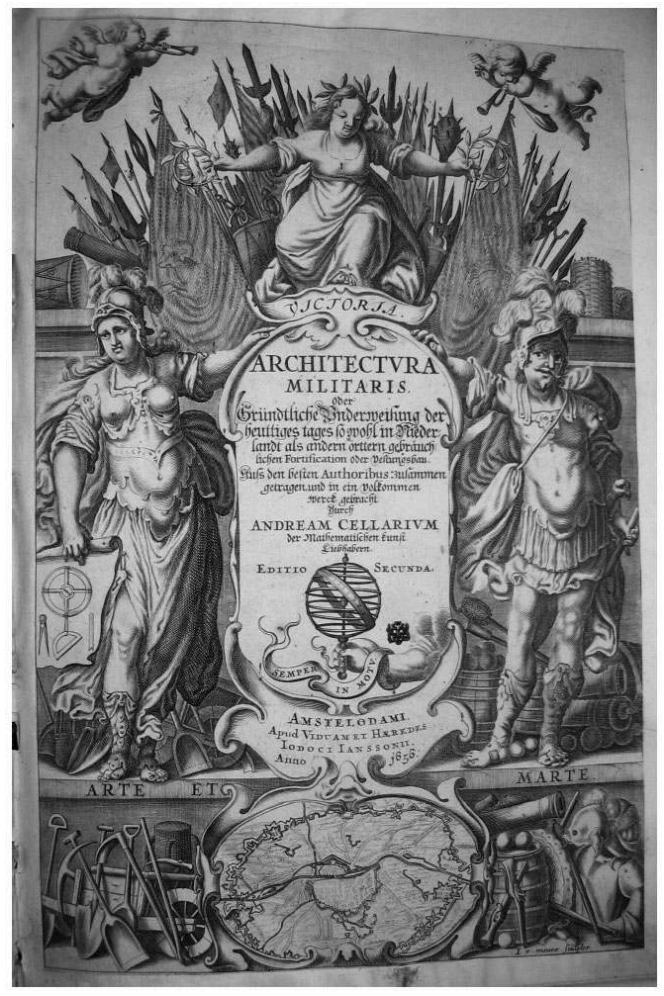

Frontispice d'Andreas Cellarius, Architectura militaris Oder Gründtliche Underweisung der heuttiges tages so wohl in Nieder, Amsterdam : Johan Jansson, 1656, BAN NIORK, 5078 f. 1847.

3

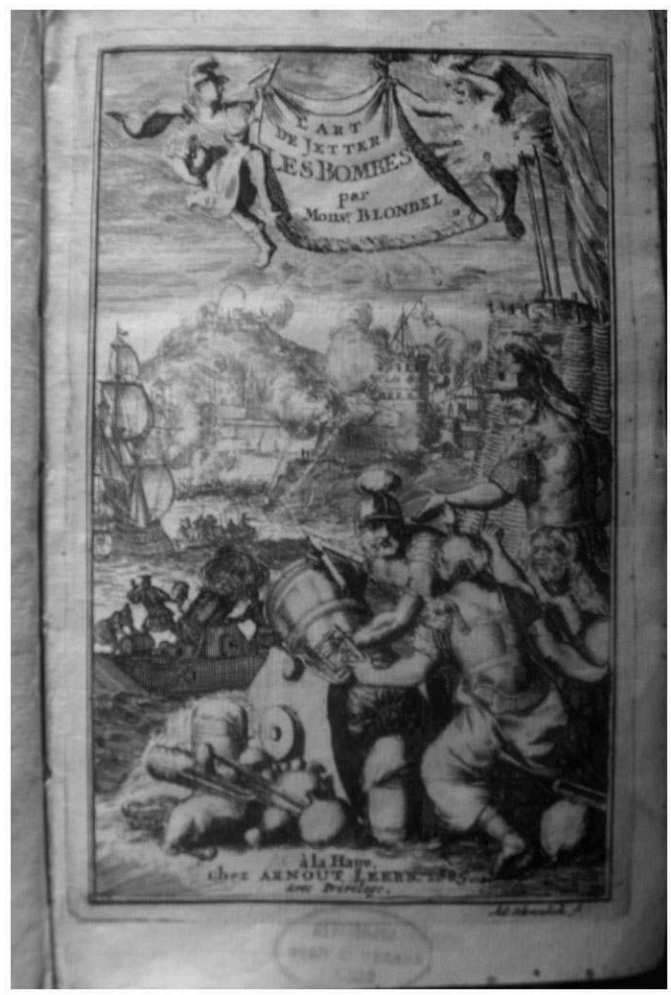

Page titre de L'Art de jetter les bombes de Blondel, La Haye : Arnout Leers, 1685, BAN OR P I, nº 46. 


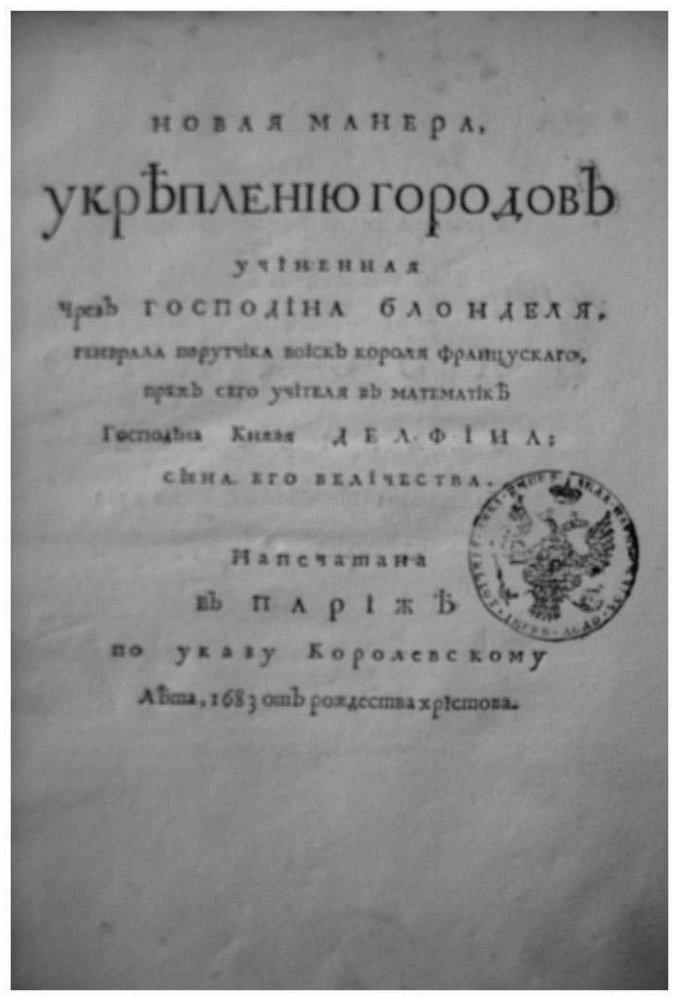

Page titre de l'ouvrage de François Blondel, Novaja manera ukrepleniju gorodov [Nouvelle manière de fortifier les places], traduction de La Nouvelle Fortification de Blondel, [traduction Ivan Zotov], Moscou, [se], 1711, BAN OR P I, nº 98

Cette volonté de Pierre le Grand d'inscrire sa bibliothèque dans l'actualité était déjà perceptible dans la composition de ses atlas manuscrits dont une récente étude a constitué un révélateur tant de sa personnalité que de ses priorités en matière d'information stratégique ${ }^{40}$. Or, il convient de se rappeler combien la constitution de cet ensemble, dessins, plans manuscrits et livres imprimés, a découlé d'une volonté précise. Il s'est agi pour le tsar de se nourrir de la plus large somme possible de références et de modèles contemporains dans des domaines et des aires géographiques qui l'intéressaient. La composition de sa bibliothèque de militaria conçue comme une galerie d'actualité architecturale et stratégique va dans le même sens. Il faut également souligner son intérêt pour la traduction de biographies antiques, comme celles d'Alexandre ou de César. Cette volonté de traduction éclaire la personnalité du tsar pour lequel les hauts faits d'armes des généraux romains étaient aussi importants que les traités d'actualité. La composition, même lacunaire, de sa bibliothèque met en valeur sa vocation de documentation princeps utile à son propriétaire et propre à servir ses intérêts et la grande entreprise de création d'un empire dans laquelle Pierre le Grand s'était engagé. Il convient cependant de nuancer cette dernière conclusion en rappelant que certains ouvrages, tel l'exemplaire de Quintus Curtius Rufus, se trouvent aujourd'hui dans le fonds russe et en soulignant que Pierre le Grand avait toujours la possibilité de consulter les bibliothèques de ses proches, qui pouvaient être plus riches et plus classiques que la sienne. 


\section{NOTES}

1. Roberto Valturio, De Re Militari, Veronae : Johannes ex Veronae impressit, 1472.

2. C. Bec, éd., Niccolo Machiavelli, Cuvres, P. : Robert Laffont, 1996; Raimondo Luraghi, éd., Le Opere di Raimondo Montecuccoli, Rome: éditions Stato maggiore dell esercito, Ufficio storico, 1988, 2 vol. ; Guillaume Du Bellay, Discipline militaire, P. : B. Rigaud, 1592.

3. Voir à ce sujet Michael E. Mallett, «Some Notes on a Fifteenth Century Condottiere and his Library: Count Antonio da Marsciano ", in C.H. Clough, éd., Cultural Aspects of the Italian Renaissance: Essays in Honour of P.O. Kristeller, New York-Manchester : Manchester University Press, 1976, p. 202-215.

4. Francesco Patrizi, Paralleli militari di Francesco Patrizi ne'quali si fa paragone delle Milizie antiche, in tutte le parti loro, con le moderne, Roma: Apresso Luigi Zanetti, 1564, vol. II, p. 205. Cité par Frédérique Verrier, Les Armes de Minerve: L'humanisme militaire dans l'Italie du XVI siècle, P. : Presses universitaires de la Sorbonne, 1997, p. 105.

5. Le sujet des réformes et de la révolution militaire initiées par Pierre le Grand est notamment abordé dans Marshall Poe, « The Consequences of the Military Revolution in Muscovy: A Comparative Perspective ", Comparative Studies in Society and History, 38 (4), 1996, p. 603-618 ; " The Military Revolution: Administrative Development, and Cultural Change in Early Modern Russia ", The Journal of Early Modern History, 2 (3), 1998, p. 247-273 ; Carol B. Stevens, « Modernizing the Military: Peter the Great and Military Reform ", in Jarmo Kotilaine et Marshall Poe, éds., Modernizing Muscovy: Reform and Social Change in Seventeenth-Century Russia, Londres: Routledge Curzon, 2004, p. 247-262. Je remercie le relecteur attentif de mon article qui a généreusement partagé ses références.

6. Olga Medvedkova, "La bibliothèque d'architecture de Pierre le Grand ", Cahiers du Monde russe, 47 (3), 2006, p. 467-502 ; Irina Beljaeva, Irina Lebedeva, "L'histoire de la bibliothèque de Pierre le Grand et de ses catalogues », ibid., p. 459-466.

7. Cf. Baljaeva, Lebedeva « L'histoire de la bibliothèque de Pierre le Grand...».

8. L.N. Puškarev, Obščestvenno-političeskaja mysl' Rossii: Vtoraja polovina XVII veka: Očerki [La pensée politique et sociale dans la Russie de la seconde moitié du XVII e siècle: Essais], M. : Nauka, 1982.

9. I.N. Lebedeva, Biblioteka Petra I: Opisanie rukopisnyh knig [La bibliothèque de Pierre I ${ }^{\text {er }}$ : description des manuscrits], SPb. : Bibliothèque de l'Académie des sciences, 2003.

10. Verrier, Les Armes de Minerve.

11. Kratkoe iz"jatie voinskih dejstv Julija Cezarja.

12. RGADA (Rossijskij gosudarstvennyj arhiv drevnih aktov - Archives d'État de Russie des actes anciens), f. 9, Cabinet de Pierre le Grand, otd. II, kn. 26, Lettres entrantes 1716 : A$Z$.

13. Aleksandrika, serbskaja redakcija, SPb.: BAN OR P I (Biblioteka Akademii nauk, otdel rukopisej, Petr I - Bibliothèque de l'Académie des sciences, Département des manuscrits, collection de Pierre $1^{\text {er }}$ ), série B, 1675-1676, nº 99.

14. Kvint Kurcij Ruf (Quinte Curce), O dejanijah Aleksandra Makedonskogo (Historiarum Alexandri Magni), traduit du latin par Il'ja Kopievskij, manuscrit, fin du XVII ${ }^{e}$ siècle. SPb. : 
BAN OR P I, série $B, n^{\circ} 81$. Cette traduction a été réalisée pour l'éditeur d'Amsterdam Jan Tessing qui travaillait sur la commande russe. Voir Lebedeva, Biblioteka Petra I, p. 191-193.

15. Caesar Cajus Julius, Rerum gestarum commentarii XIV, Lugduni [Lyon], Apud Antonium de Harst, 1612, BAN OR PI, $\mathrm{n}^{\circ} 37$.

16. Ju.N. Bespjatyh, Inostrannye istočniki po istorii Rossii pervoj četverti xVIII v. (C. Uitvort, G. Grund, L.Ju. Erenmal'm), [Les sources étrangères sur l'histoire de la Russie du premier quart du XVIII siècle), SPb., 1998, p. 363; voir aussi : P.A. Krotov, "Polkovodčeskoe iskusstvo Petra I i A.D. Menšikova v Poltavskoj bitve (K 300-letiju Poltavskoj bitvy)» [L'art de stratège : Pierre le Grand et A.D. Menšikov dans la bataille de Poltava (pour le $300^{\mathrm{e}}$ anniversaire de la bataille)], in Menšikovskie čtenija, SPb., 2007, p. 5.

17. Flavius Josèphe, La guerre des juifs, version manuscrite. SPb. : BAN OR P I, $n^{\circ} 76$.

18. Aelianus Tacticus, De militaribus ordinibus instituendis more grcecorum liber à Francisco Robortello, Venise : Andrea et Jacobum Spinellos, 1552., BAN NIORK (Naučno-issledovatel 'skij otdel redkoj knigi - Département des livres rares scientifiques), 8011q/338R.

19. Sébastien Le Prestre de Vauban, Istinnyj sposob ukreplenija gorodov, izdannyj ot slavnogo inženera Vobana na francuzskom jazyke. Nyne že pereložen s francuzskogo jazyka na rossiiskji jazyk. Perevel V.V. Suvorov [Bonne manière de fortifier les villes publiée par le célèbre ingénieur Vauban en langue française et maintenant traduit en russe par V. Suvorov], SPb. : imprimerie de Saint-Pétersbourg, 1724.

20. Allain Manesson Mallet, Les travaux de Mars, ou l'art de la guerre. Divisez en trois parties, Amsterdam : Henri Desbordes, 1696, BAN OR P I, nº 89.

21. Manesson Mallet, Les Travaux de Mars, P.: Denis Thierry, 1685, vol. 2, p. 161.

22. Jean Charles de Folard parfois appelé "Le Végèce français » cité par Jean-Jacques Langendorf, "L'humanisme de l'officier frédéricien et post-frédéricien", in Bruno Colson, éd., Pensée stratégique et humanisme, de la tactique des anciens à l'éthique de la stratégie, P. : Economica, 2000, p. 177.

23. Buonaiuto Lorini, Le fortificationi di Buonaiuto Lorini, nobile Fiorentino, Venetia: Presso Francesco Rampazetto, 1609, BAN OR P I, n 405 ; Lorini Buonaiuto, Das sechste Buch Von der Fortification, [s.e], 1616, BAN OR P I, nº 420.

24. Wilhelm Dilich, Verschiedener Churund Fuersten gewesenen weitberuehmten Ingenieurs, Francfort-sur-le-Main, chez Iohann David Zunners et Iohann Philipp Andreæ, 1689, BAN OR P I, $n^{\circ} 406$.

25. Adam Freitag, L'architecture militaire ou la fortification nouvelle, P. : Toussainct Quinet, 1640, BAN OR P I, nº 417.

26. Ambroise Bachot, Le gouvernail... lequel conduira le curieux de géométrie en perspective dedans l'architecture des fortifications (...), Melun-Paris : l'auteur, 1592, BAN OR P I, nº 407.

27. Georg-Andreas Böckler, Manuale architecture militaris, Franckfurt: Thomas-Matthia Goetzen, 1645, BAN OR P I, n 127.

28. Wendelin Schildknecht, Harmonia In Fortalitiis construendis, defendendis \& oppugnandis, Stettin : Gedruckt und verlegt von Johann Valentin Rheten, 1652, BAN OR P I, n 411.

29. Andreas Cellarius, Architectura militaris Oder Gründtliche Underweisung der heuttiges tages so wohl in Nieder, Amsterdam : Johan Jansson, 1656, BAN NIORK, 5078 f. 1847.

30. Antoine De Ville, De la charge des gouverneurs des places..., P.: Compagnie des marchands libraires du Palais, 1666, BAN OR P I, nº 78; A De Ville, Die Festungs-Bau-Kunst 
oder der vollkommene Ingenieur (...), Amsterdam: Abraham Wolffgangs Hendrich et Dietrich Boom, 1676, BAN OR P I, $\mathrm{n}^{\circ} 71$.

31. François Blondel, L'Art de jetter les Bombes par Mons ${ }^{r}$ Blondel, La Haye : Arnout Leers, 1685, BAN OR P I, $\mathrm{n}^{\circ} 46$.

32. Menno van Coehorn, Nouvelle Fortification, tant pour un Terrain bas et humide que sec et élevé, Amsterdam : Jan Wesel, 1706, BAN OR P I, n 70.

33. Johann Jacob Werdmüller, Des Commendanten-Spiegel, oder Gruendlicher Unterrichtung, wie ein Commandant seinen Platz forticieren, verbessern in gutem Stand erhalten sole, Franckfurt a. M. : druckts Joh. Goerlin, 1685, BAN OR P I, n 79.

34. Johann-Bernhard Scheiter, Examen fortificatorium, Darin so wohl Eine gantz newe Art oder Manier vom Vestungs, Straszburg: Bey Johann Fridriech Spoor und Reinhard Waechtler, 1676, BAN OR P I, nº 408.

35. Pierre Surirey de Saint-Remy, Mémoires d'artillerie, Recueillis par $M^{r}$ Surirey de Saint Remy, Lieutenant du Grand Maistre de l'Artillerie de France, P.: Ricaud, Directeur de l'Imprimerie Royale, rue de la Harpe, 1707. 2 vol., BAN OR P I, n 19.

36. Chevalier de Saint-Julien, Architecture militaire, ou l'Art de fortifier les villes, La Haye: Jean van Millinge, 1705, BAN OR P I, n 68 ; Chevalier de Saint-Julien, La forge de Vulcain, ou l'appareil des machines de guerre, La Haye : Guillaume de Voys, 1706, BAN OR P I, nº 69.

37. Sieur de Goulon, Mémoires pour l'attaque et pour la défense d'une place, Wesel, [s.e], 1706, BAN OR P I, $\mathrm{n}^{\circ} 60$.

38. Leonhard-Christoph Sturm, Le véritable Vauban se montrant au lieu du faux Vauban, La Haye: 1708, BAN OR PI, $\mathrm{n}^{\circ} 22$; Leonhard-Christoph Sturm, Architectura militaris Hypothetica et Eclectican, Nüremberg, [se], 1702, BAN OR P I, nº 80.

39. Atlas portatif ou le Théâtre de la guerre en Europe. Contenant les cartes géographiques avec le plan des villes \& forteresses les plus exposées aux révolutions présentes, Amsterdam : Daniel de la Feuille, 1702, BAN OR P I, n ${ }^{\circ} 88$; Nicolas De Fer, Les forces de l'Europe, ou description des principales villes avec leurs fortifications, P. : chez l'auteur, 1793-1796, BAN OR P I, nº 371.

40. Émilie d'Orgeix, «La collection du Prince artisan: Les dessins de fortifications de Pierre le Grand conservés à l'Académie des sciences de Saint-Pétersbourg ", Cahiers du Monde russe, 47 (3), 2006, p. 503-514.

\section{RÉSUMÉS}

Résumé

La composition de la bibliothèque du prince moderne, miroir de ses penchants, de ses aspirations sociales et politiques, de son dilettantisme, de sa volonté de spécialisation, de son intérêt pour l'actualité, trouve un écho particulier dans le choix de ses ouvrages militaires. L'équilibre entre auteurs anciens et modernes, entre poliorcétique antique et architecture bastionnée, révèle plus encore que le goût de leur propriétaire, l'expression de sa modernité. C'est dans cette perspective que cet article se penche sur la composition de la bibliothèque de militaria de Pierre le Grand, Elle permet d'éclairer l'attitude d'un souverain, féru d'art et d'antiquités, envers l'art 
militaire, de découvrir les lectures qu'il a privilégiées et les guides littéraires qui ont accompagné la construction de la plus grande ville fortifiée ex nihilo du XVIII ${ }^{\mathrm{e}}$ siècle dans un siècle traversé par le grand bouleversement baroque.

Military science books from Peter the Great's library

The contents of a modern prince's library reflect his inclinations, social and political aspirations, dilettantism, desire for specialized knowledge, and interest in current events. The prince's choice of military texts is a good illustration of this. The balance between ancient and modern authors, between antique siege warfare and fortifications reveals, beyond the taste of the collector, the expression of his modernity. This constitutes the framework of this article, which focuses on the collection of Militaria in Peter the Great's library and sheds light on the attitude of this lover of art and antiquities towards military arts. The article also presents Peter's favorite texts and the literary models that accompanied the construction ex nihilo of the largest fortified city of the eighteenth century - a century that was marked by the great Baroque upheaval.

\section{AUTEUR}

\section{ÉMILIE D'ORGEIX}

Institut national d'histoire de l'art, ParisUniversité Michel de Montaigne - Bordeaux 3 